\title{
Brote de escabiosis en el Hospital Víctor Lazarte Echegaray, Trujillo, 2017
}

\author{
Marco Antonio Alfaro Angulo* ${ }^{\text {; }}$ Ana Maria Ruth Canevaro Alva ${ }^{2}$
}

RESUMEN

Objetivo: Describir las características de un brote de escabiosis y evaluar el impacto de las medidas de control.

Materiales y métodos: Estudio epidemiológico descriptivo y prospectivo, realizado en un hospital de referencia, con 220 camas de hospitalización. Los datos se recolectaron a través de visitas diarias al Servicio de Medicina, entrevistas al personal asistencial y revisión de las historias clínicas. En la descripción del brote se consideran las variables epidemiológicas de persona, lugar y tiempo. Se aplicó estadística descriptiva, y se determinaron las frecuencias absolutas y relativas y la tasa de ataque. Se solicitó consentimiento informado a los pacientes y al personal asistencial, se les explicó sobre el beneficio de la investigación. La intervención consistió en medidas de aislamiento y precauciones de transmisión por contacto, se enfatizó el uso de guantes no estériles.

Resultados: El brote ocurrió desde la semana epidemiológica 29 hasta la 37 del 2017. El caso primario fue un paciente varón de 93 años de edad con sarna noruega, hospitalizado por demencia de Alzheimer. El cuadro se presentó en 9 enfermeras, 7 médicos residentes, 6 técnicos de enfermería, 1 interno de medicina y 5 pacientes hospitalizados.

Conclusiones: El brote fue de fuente propagada, con duración de 9 semanas epidemiológicas; y afectó enfermeras, médicos residentes, internos de medicina y a pacientes hospitalizados. La tasa de ataque fue del $40 \%$. Las medidas de prevención y control fueron aislamiento del caso primario y de los pacientes, higiene de manos antes y después de la atención, uso de guantes no estériles, uso de bata, artículos de uso clínico exclusivos para cada paciente, la ropa de los pacientes con sarna fue manipulada con guantes y en bolsas cerradas, limpieza de superficies, exclusión de la atención clínica de los trabajadores de la salud con sarna hasta las 24 horas de iniciado el tratamiento, y la vigilancia epidemiológica. El tratamiento médico consistió en loción de permetrina al $5 \%$ por tres días. Se recomienda aplicar normas de bioseguridad en la atención de todos los pacientes, sobre todo cuando se hospitalizan con lesiones dermatológicas. Las medidas que se implementaron deben tenerse en cuenta en la prevención y control de este tipo de brote.

Palabras clave: Brotes de enfermedades; Escabiosis; Personal de salud (Fuente DeCS BIREME).

\section{Scabies outbreak at the Hospital Víctor Lazarte Echegaray, Trujillo, 2017}

\section{ABSTRACT}

Objective: To describe the characteristics of a scabies outbreak and assess the effect of the control measures.

Materials and methods: A descriptive and prospective epidemiological study was conducted at a reference hospital with 220 inpatient beds. Data was collected by visiting the Medicine Service on a daily basis, interviewing the healthcare personnel and examining the medical records. The description of the outbreak includes epidemiological variables such as subject, place and time. Descriptive statistics was used to determine the absolute and relative frequencies, as well as the attack rate. Patients and healthcare personnel were requested to sign an informed consent and received information about the benefits of the research. The intervention consisted of isolation measures and contact transmission-based precautions. The use of non-sterile gloves was emphasized.

Results: The outbreak took place between the epidemiological weeks 29 and 37 in the year 2017. The index case was a 93-year-old male patient who had Norwegian scabies and was admitted to the hospital due to Alzheimer's disease. The scabies occurred in nine female nurses, seven residents, six nurse technicians, one intern y five inpatients.

Conclusions: This propagated-source outbreak lasted nine epidemiological weeks; and affected nurses, residents, interns and inpatients. The attack rate was $40 \%$. The prevention and control measures were isolation of the index case and patients, hand hygiene before and after patient care, use of non-sterile gloves, use of scrubs, use of individual medical supplies for each patient, handling of clothing from scabies-infected patients in sealed bags and using gloves, surface cleaning, withdrawal of scabies-infected health workers from their duties until 24 hours of treatment onset, and epidemiological surveillance. Medical treatment was $5 \%$ permethrin lotion for three days. Use of biosafety standards is recommended for patient care, especially when patients are admitted to the hospital with skin lesions. The implemented measures must be considered to prevent and control this type of outbreak.

Keywords: Disease outbreaks; Scabies; Health personnel (Source: MeSH NLM).

1. Red Asistencial La Liberad, EsSALUD. La Libertad, Perú.

2. Hospital I Luis Albrecht, EsSALUD. La Libertad, Perú.

* Autor corresponsal 


\section{INTRODUCCIÓN}

La escabiosis o sarna es la infestación de la piel por el Sarcoptes scabiei variedad hominis; el nombre Sarcoptes se deriva de la palabra griega sarx, que significa carne, y la palabra koptein, que significa cortar. El término Scabiei proviene de la palabra latina scabere, que quiere decir arañar. Aristóteles fue el primero en usar la palabra Akari al mencionar un ácaro del bosque, que se describió hace 2500 años, hasta que, en 1687, Bonono y Cestoni identificaron el organismo y lo asociaron con las lesiones cutáneas ${ }^{(1-4)}$.

La sarna es endémica; sin embargo, a lo largo de la historia se han descrito brotes cada 20 a 30 años. Ocurren en otoño e invierno y el hacinamiento durante el invierno favorece la trasmisión ${ }^{(5)}$. Es una enfermedad prevalente en todo el mundo, sobre todo en países en vías de desarrollo ${ }^{(6,7)}$, con una incidencia de 300 millones de casos cada año; generalmente, afecta a niños que viven en zonas tropicales pobres y sobrepobladas ${ }^{(6)}$. La distribución en las poblaciones más jóvenes probablemente refleja mayor exposición al parásito e inadecuada inmunidad del hospedador. La afección no distingue sexo y las diferencias étnicas se relacionan con el nivel socioeconómico, la pobreza, falta de vivienda o vivienda hacinada, el estado nutricional deficiente, demencia, higiene deficiente y factores de comportamiento ${ }^{(8,9)}$.

El ácaro se transmite por contacto directo, piel con piel, con una persona enferma. Se contagia fácilmente a los miembros de la familia, a las parejas sexuales (si el contacto es por lo menos de diez minutos) y, a veces, se propaga indirectamente al compartir artículos como ropa, toallas o ropa de cama usada por una persona infestada. Puede ocurrir más fácilmente en el caso de la sarna noruega. Un rápido apretón de manos o un abrazo no propagan la enfermedad (10-13).

La transmisión ocurre cuando los ácaros femeninos recién apareados penetran la epidermis por la secreción de una solución que disuelve la piel (12). Las hembras adultas fecundadas penetran el estrato córneo, donde ponen un promedio de 0 a 4 huevos por día durante, aproximadamente, 2 meses; menos del $10 \%$ de estos se convertirán en adultos en 2 semanas. Una vez que los ácaros alcanzan la etapa adulta, abandonan sus madrigueras y emergen a la superficie de la piel, donde se aparean, y repiten el ciclo de vida. Por otro lado, una persona infestada puede transmitir, incluso sin presentar síntomas, hasta que sea tratado con éxito ${ }^{(10,14,15)}$.
El período de incubación es de 2 a 6 semanas, si una persona ha tenido la enfermedad, los síntomas aparecen de 1 a 4 días después de la exposición. En la sarna normal, los signos y síntomas más comunes de la enfermedad son prurito intenso, especialmente, por la noche y pápulas en la piel, que pueden afectar gran parte del cuerpo o limitarse a sitios comunes como la muñeca, el codo, la axila, entre los dedos, el pezón, el pene, la cintura y las nalgas. La erupción puede ir acompañada de vesículas y escamas. A veces, se observan pequeñas madrigueras en la piel, formadas por el túnel que producen los ácaros hembras, que consisten en diminutas líneas en relieve y torcidas (serpiginosas) de color blanco grisáceo o de color de la piel. La cabeza, la cara, el cuello, las palmas de las manos y las plantas de los pies a menudo están afectadas en niños muy pequeños ${ }^{(10,16)}$.

Existe otra forma de presentación denominada sarna noruega, que debe su nombre a la primera descripción de la enfermedad en pacientes leprosos de Noruega, en 1848 . Se caracteriza por una hiperqueratosis de la epidermis, con costras grisáceas, gruesas y adherentes. Las lesiones se ubican en las superficies flexoras, dorso, cabeza, región retroauricular y en zona palmar y plantar. Hay compromiso del lecho ungueal de manos y pies con engrosamiento de las uñas, descamación de la piel, que incluso puede ser generalizada y provocar un cuadro de eritrodermia, habitualmente, sin prurito. La presentación se relaciona con una disminución de la respuesta humoral de células $T$, como ocurre en los pacientes que viven con $\mathrm{VIH}$, infección por HTLV1, tratamientos inmunosupresores, radioterapia, quimioterapia o en defectos congénitos de la respuesta inmune ${ }^{(17,18)}$.

Si bien la contagiosidad de la sarna normal se considera baja, un mayor riesgo de transmisión se asocia con los casos de sarna noruega, altamente infectante, ya que es posible encontrar millones de ácaros en las lesiones costrosas. Si el paciente se encuentra hospitalizado puede contagiar al personal de salud y a los pacientes, y ocasionar brotes ${ }^{(10,18,19)}$.

El diagnóstico, generalmente, se basa en la apariencia y distribución habituales de la erupción y la presencia de madriguera. Para la confirmación es necesario identificar a los ácaros, su materia fecal o los huevos (10).

El prurito intenso ocasiona rascado que lesiona la piel, susceptible de infectarse con bacterias, como Staphylococcus aureus o estreptococos 
betahemolíticos. Se describe que la infección bacteriana de la piel puede originar glomerulonefritis postestreptocócica, fiebre reumática y bacteriemia. En la sarna noruega, comúnmente, no se presenta el prurito ${ }^{(10,16,20-24)}$.

La sarna, por lo general, se presenta en la comunidad, inclusive, con brotes en grupos familiares y sus mascotas (25); cuando ocurre en los establecimientos de salud se originan brotes como el que se reporta. El estudio describe la transmisión intrahospitalaria de esta parasitosis, y tiene como objetivos describir las características del brote de escabiosis y evaluar las medidas de control.

\section{MATERIALES Y MÉTODOS}

\section{Diseño y población}

Estudio epidemiológico descriptivo y prospectivo, realizado en el Hospital Víctor Lazarte Echegaray, de la ciudad de Trujillo, Perú. Es un establecimiento de salud de referencia con 220 camas de hospitalización. El Servicio de Medicina donde ocurrió el brote tiene 75 camas, el $34 \%$ del total de camas del hospital.

Los datos se recolectaron a través de visitas diarias al Servicio de Medicina, entrevistas al personal asistencial y revisión de historias clínicas. Se consideró como caso sospechoso al personal de salud que labora en el servicio y a los pacientes hospitalizados, con antecedente de haber estado en contacto con el caso primario o haberlo atendido, que desarrollaron signos o síntomas de escabiosis y, como caso confirmado, a todo caso sospechoso con evaluación de un dermatólogo o internista o infectólogo con o sin confirmación parasitológica.

\section{Variables de estudio}

Al ser la comunicación de un brote, se consideraron las variables epidemiológicas de persona, lugar y tiempo.

La intervención consistió en la implementación de varias medidas. El caso primario se aisló en una habitación individual con puerta cerrada y con precauciones de transmisión por contacto hasta el alta, y los pacientes hospitalizados con sarna se aislaron en cohorte. Las precauciones de contacto incluyeron higiene de manos antes y después de la atención. Se enfatizó el uso de bata y de guantes no estériles ${ }^{(16)}$ al entrar en contacto con el paciente. Los artículos de uso clínico (manguito de presión, termómetro, etc.) fueron de uso exclusivo para cada paciente. Los trabajadores de la salud con sarna fueron excluidos de la atención clínica hasta las 24 horas de iniciado el tratamiento. La ropa de los pacientes con sarna fue manipulada con guantes y en bolsas cerradas; el aseo de las habitaciones y la limpieza de superficies se realizaron de acuerdo con las normas. El tratamiento consistió en loción de permetrina al $5 \%$ por tres días e implementación de vigilancia epidemiológica ${ }^{(22,26-31)}$.

La vigilancia epidemiológica se mantuvo hasta seis semanas después de la hospitalización del caso primario y consistió en registro de casos, entrevistas al personal asistencial que atendieron al paciente con sarna noruega, indagar acerca de signos o síntomas compatibles con sarna y búsqueda de casos atípicos o no reconocidos. Desde el punto de vista organizativo, se realizó reunión con el director del hospital y jefes de servicio para informar acerca del brote y dar a conocer las medidas de control. Se explicó al personal del servicio los mecanismos de transmisión de la enfermedad y las medidas de control.

\section{Análisis estadístico}

Para tratar las variables epidemiológicas se utilizaron frecuencias absolutas y relativas, y se calculó la tasa de ataque.

\section{Consideraciones éticas}

Se solicitó consentimiento informado a los pacientes y al personal asistencial, y se les explicó sobre el beneficio de la investigación, es decir, evitar la propagación.

\section{RESULTADOS}

El brote inició en la semana epidemiológica N. 29 y finalizó en la 37 del año 2017 (Figura 1). El caso primario fue un paciente varón, de 93 años de edad, con diagnóstico de ingreso de demencia por Alzheimer. La sarna noruega se diagnosticó después de cinco días de hospitalización. Los casos secundarios en el personal de salud y en los pacientes internados se detectaron a los 7 días de contacto con el caso primario y los síntomas se presentaron en un rango de 7 a 13 días. 


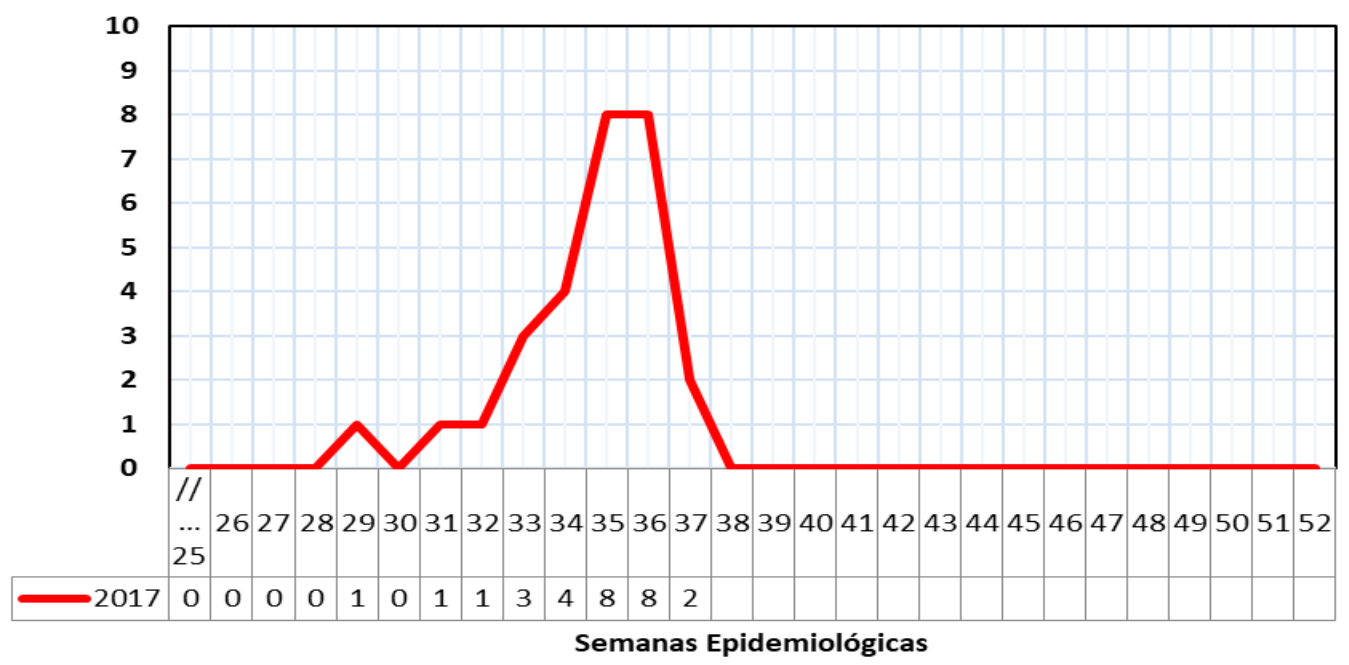

Figura 1. Curva epidémica de escabiosis. Hospital Víctor Lazarte Echegaray, 2017

El brote afectó a 9 enfermeras, 7 médicos residentes, 6 técnicos de enfermería y un interno de medicina (Tabla 1). Además, 5 pacientes hospitalizados ( 3 hombres y 2 mujeres) fueron contagiados por el personal de salud, que no habían usado guantes al atender al paciente con sarna noruega, según informaron en la entrevista. La tasa de tasa de ataque fue de $40 \%$.

Tabla 1. Personal de salud y pacientes afectados con escabiosis. Hospital Víctor Lazarte Echegaray, 2017

\begin{tabular}{|lcl}
\hline \multicolumn{2}{c}{ Población afectada por brote de escabiosis } \\
\hline \multicolumn{1}{|c}{ Personal de salud / pacientes } & N. ${ }^{\circ}$ & $\%$ \\
\hline Enfermeras & 9 & 32 \\
\hline Médicos residentes & 7 & 25 \\
\hline Técnicos de enfermería & 6 & 21 \\
\hline Pacientes & 5 & 18 \\
\hline Internos de Medicina & 1 & 4 \\
\hline Total & 28 & 100 \\
\hline
\end{tabular}

\section{DISCUSIÓN}

El brote se inició cuando un paciente de 93 años es hospitalizado en el Servicio de Medicina, con diagnóstico de ingreso de demencia por Alzheimer y con lesiones en la piel que hicieron sospechar dermatitis exfoliativa, lo que motivó retardo del diagnóstico de escabiosis. Esta situación es descrita por varios autores que mencionan que el diagnóstico se hace más difícil en pacientes de mayor edad, y en inmunocomprometidos porque presentan síntomas atípicos o lesiones inespecíficas ${ }^{(3,16,19,21)}$.

En los brotes de esta enfermedad influyen diversos factores. Comúnmente, si un paciente con sarna noruega es hospitalizado y pasa inadvertido; el diagnóstico demora y el personal tiene contacto físico sin emplear guantes, como se evidencia en el brote que describimos. Por lo tanto, es recomendable implementar medidas de prevención al hospitalizar pacientes con enfermedades dermatológicas $(19,25-27)$. La sarna noruega es un factor que favorece la 
ocurrencia de brotes de escabiosis en la hospitalización (3).

El brote se originó de una fuente propagada (Figura 1), característica inherente a la diseminación de la enfermedad. Los casos secundarios de sarna se observaron, principalmente, en enfermeras y médicos residentes que laboran en el Servicio de Medicina y en algunos pacientes hospitalizados (Tabla 1). Las enfermeras y los técnicos de enfermería son los más afectados debido a que mantienen contacto más cercano y prolongado con los pacientes $(30,31)$. La mayoría del personal asistencial mostró poca adherencia al empleo de guantes, lo que concuerda con otras investigaciones; el uso de guantes no estériles es muy importante en el control de la transmisión, pues la higiene de manos no es suficiente, ya que los ácaros de la sarna son resistentes al agua, al jabón y a soluciones con alcohol ${ }^{(29,30)}$.

Los síntomas se iniciaron entre los días 7 a 13, es decir, que el tiempo puede variar entre las 24 horas y las seis semanas (3), de acuerdo a la reacción inmunológica del paciente. La tasa de ataque fue $40 \%$, otros estudios han documentado tasas que varían del 4,1 al $37 \%$ (19,31-36). Este parámetro es un indicador importante para describir los brotes porque mide la ocurrencia de casos durante su curso, relaciona el número de casos con la población expuesta al riesgo, que, en este caso, está constituida por el personal de salud y los pacientes hospitalizados ${ }^{(37)}$.

En conclusión, el brote de escabiosis se originó en un paciente del Servicio de Medicina (fuente propagada), se trasmitió de persona a persona, dentro del personal afectado estuvieron médicos residentes, enfermeras, técnicos de enfermería, internos de medicina y también otros pacientes hospitalizados. El brote se extendió por 9 semanas con una tasa de ataque del $40 \%$. Las medidas de prevención y control incluyeron aislamientos de los pacientes, higiene de manos antes y después de la atención, uso de guantes no estériles y de bata, manipulación de ropa de pacientes con sarna en bolsas cerradas, limpieza de las superficies y exclusión de la atención clínica de los trabajadores de la salud con sarna hasta las 24 horas de iniciado el tratamiento; además de la vigilancia epidemiológica. Como tratamiento se empleó la permetrina al $5 \%$. Se recomienda aplicar normas de bioseguridad durante la atención de todos los pacientes, especialmente, si se presentan lesiones dermatológicas; además, se deben tener en cuenta las medidas preventivas que se implementaron en este trabajo.

\section{REFERENCIAS BIBLIOGRÁFICAS}

1. Montesu MA, Cottoni F. G. C. Bonomo and D. Cestoni. Discoverers of the parasitic origin of scabies. Am J Dermatopathol. 1991; 13(4): 425-7.
2. Van Neste DJJ. Inmunology of scabies. Parasitol Today. 1986; 2(7): 194-6.

3. Walton SF, Currie BJ. Problems in diagnosing scabies, a global disease in human and animal populations. Clin Microbiol Rev. 2007; 20(2): 268-79.

4. Heukelbach J, Feldmeier H. Scabies. The Lancet. 2006; 367(9524): 1767-74.

5. Green MS. Epidemiology of scabies. Epidemiol Rev. 1989; 11: 126-50.

6. Andrews RM, McCarthy J, Carapetis JR, Currie BJ. Skin disorders, including pyoderma, scabies and tinea infections. Pediatr Clin North Am. 2009; 56(6): 1421-40.

7. Chosidow O. Scabies. N Engl J Med. 2006; 354(16): 1718-27.

8. Tsutsumi M, Nishiura H, Kobayashi T. Dementia-specific risks of scabies: retrospective epidemiologic analysis of an unveiled nosocomial outbreak in Japan from 1989-90. BMC Infect Dis. 2005; 5: 85.

9. Makigami K, Ohtaki N, Ishii N, Tamashiro T, Yoshida S, Yasumura S. Risk factors for recurrence of scabies: a retrospective study of scabies patients in a long-term care hospital. J Dermatol. 2001; 38(9): 874-9.

10. Centers for Disease Control and Prevention. Scabies [Internet]; 2018. Disponible en: https://www.cdc.gov/ parasites/scabies/gen_info/faqs.html

11. Fain A. Epidemiological problems of scabies. Int J Dermatol. 1978; 17(1): 20-30.

12. Laliena Aznar S, Corella Aznar E, Campos Calleja C, Gómez Barrena V. Escabiosis: lesiones pruriginosas altamente contagiosas. Rev Ped Elec. 2018; 15(2): 31-6.

13. Sola García E, Blasco Morente G. Escabiosis: la dermatoscopia puede ser la clave. Actual Med. 2017; 102(802): 178-9.

14. Arlian LG, Runyan RA, Achar S, Estes SA. Survival and infectivity of Sarcoptes scabiei var. canis and var. hominis. J Am Acad Dermatol. 1984; 11(2Pt1): 210-5.

15. Ministerio de Salud del Perú. Escabiosis. Oficina General de Tecnologías de la Información [Internet]. Lima: Minsa; 2017. Disponible en: http: //www.minsa.gob.pe

16. Leistner R, Buchwald D, Beyer M, Philipp S. Scabies outbreak among healthcare workers in a German acute care hospital. J Infect Prev. 2017; 18(4): 189-92.

17. Campos B, Jofré L, Neira P, Noemi I, Saavedra T, San Martín A. Guía clínica contra sarna y pediculosis. Ministerio de Salud: Gobierno de Chile; 2007.

18. Salavastru CM, Chosidow O, Boffa MJ, Janier M, Tiplica GS. European guideline for the management of scabies. J Eur Acad Dermatol Venereol. 2017; 31(8): 1248-53.

19. Elgueta NA, Parada EY, Guzmán GW, Molina CP, González AP. Brote de sarna en un hospital terciario a partir de un caso de sarna costrosa. Rev Chil Infectol. 2007; 24(4): 306-10.

20. Bouvresse $S$, Chosidow 0 . Scabies in healthcare settings. Curr Opin Infect Dis. 2010; 23(2): 111-8.

21. Karthikeyan K. Scabies in children. Arch Dis Child Educ Pract Ed. 2007; 92(3): 65-9.

22. Del Borgo C, Belvisi V, Tieghi T, Mastroianni CM. Atypical presentation of crusted (Norwegian) scabies. Infection. 2015; 43(5): 623-4.

23. Boureau AS, Cozic C, Poiraud C, Varin S, Chaillous B, Cormier G. Does immunodepression induced by TNF antagonists promote atypical scabies?. Joint Bone Spine. 2014; 81(2): 186-7.

24. Hay RJ, Steer AC, Engelman D, Walton S. Scabies in the 
developing world-its prevalence, complications, and management. Clin Microbiol Infect. 2012; 18(4): 313-23.

25. Gallegos JL, Budnik I, Peña A, Canales M, Concha M, López J. Sarna sarcóptica: comunicación de un brote en un grupo familiar y su mascota. Rev Chil Infectol. 2014; 31(1): 47-52.

26. Capobussi M, Sabatino G, Donadini A, Tersalvi CA, Castaldi S: Control of scabies outbreaks in an Italian Hospital: an information-centered management strategy. Am J Infect Control. 2014; 42(3): 316-20.

27. FitzGerald D, Grainger RJ, Reid A. Interventions for preventing the spread of infestation in close contacts of people with scabies. Cochrane Database Syst Rev. 2014; (2): CD009943.

28. Maestre Naranjo M, Martínez Fernández E, Fuentes Piñeiro M, Gómez Grego R, García Arcones R, Rodríguez de la Pinta ML. ¿Qué hacemos ante un brote de escabiosis en un hospital?. Med Segur Trab. 2014; 60(234): 239-43.

29. Furuya K, Nakajima H, Sasaki Y, Ishiko A, Urita Y. A scabies outbreak in a diabetic and collagen disease ward: management and prevention. Exp Ther Med. 2016; 12(6): 3711-5.

30. Hardy M, Engelman D, Steer A. Scabies: a clinical update. Aust Fam Physician. 2017; 46(5): 264-8.

31. Obasanjo 0O, Wu P, Conlon M, Karanfil LV, Pryor P, Moler G, et al. An outbreak of scabies in a teaching hospital: lessons learned. Infect Control Hosp Epidemiol. 2001; 22(1): 13-8.

32. Bellisimo-Rodrigues F, Silva MF, de Souza RP, de Oliveira e Castro Pde T. Alcohol-based hand rub and nosocomial scabies. Infect Control Hosp Epidemiol. 2008; 29(8): 782-3.

33. Khan A, O'Grady S, Muller MP. Rapid control of a scabies outbreak at tertiary care hospital without ward closure. Am J Infect Control. 2012; 40(5): 451-5.

34. Larrosa A, Cortés-Blanco M, Martínez S, Clerencia C, Urdániz LJ, Urbán J, et al. Nosocomial outbreak of scabies in a hospital in Spain. Euro Surveill. 2003; 8(10): 199-203.

35. Fajardo-Velázquez R, Urdez-Hernández E, Ysita-Morales A. Brote intrahospitalario de escabiasis, a partir de un caso de sarna noruega. Salud Pública Méx. 2004; 46(3): 251-4.

36. Larrosa Montañés A, Cortés Blanco M, Clerencia Casorrán C, Martínez Cuenca S, Urdániz Sancho L, Urbán Sender J, et al. Brote de escabiosis en una residencia para personas mayores en la provincia de Huesca. Rev Esp Salud Pública. 2004; 78(1): 107-14.

37. Isaza Nieto P. Glosario de epidemiología. 1a ed. Colombia: Academia Nacional de Medicina de Colombia; 2015.

\section{Fuentes de financiamiento:}

Este artículo ha sido financiado por los autores.

Conflicto de interés:

Los autores declaran no tener ningún conflicto de interés.

\section{Correspondencia:}

\section{Marco Antonio Alfaro Angulo}

Dirección: Urb. Juan Pablo II 2da. Etapa - E1. Trujillo, La Libertad, Perú.

Teléfono: 044287738

Correo electrónico: marcoalfaroa@yahoo.com.mx

Recibido: 15 de abril de 2019.
Evaluado: 20 de junio de 2019.
Aprobado: 29 de junio de 2019.

( La revista. Publicado por Universidad de San Martín de Porres, Perú. (cc) ${ }_{\text {BY }}$ Licencia de Creative Commons Artículo en acceso abierto bajo términos de Licencia Creative Commons Atribución 4.0 Internacional. (http://creativecommons.org/licenses/by/4.0/)

\section{ORCID iDs}

Marco Antonio Alfaro Angulo Ana Maria Ruth Canevaro Alva

https://orcid.org/0000-0002-6105-4649 https://orcid.org/0000-0002-8896-882X 